\title{
Automated volumetric segmentation method for computerized- diagnosis of pure nodular ground-glass opacity in high-resolution CT
}

\author{
Wooram Son ${ }^{a}$, Sang Joon Park ${ }^{a}$, Chang Min Park ${ }^{b}$, Jin Mo Goo ${ }^{b}$ and Jong Hyo Kim*a,b \\ ${ }^{a}$ Interdisciplinary Program in Radiation Applied Life Science, Seoul National University, \\ 101, Daehangno, Jongno-Gu, Seoul, 110-744 South Korea; \\ ${ }^{b}$ Department of Radiology, Seoul National University Hospital, \\ 101, Daehangno, Jongno-Gu, Seoul, 110-744 South Korea;
}

\begin{abstract}
While accurate diagnosis of pure nodular ground glass opacity (PNGGO) is important in order to reduce the number of unnecessary biopsies, computer-aided diagnosis of PNGGO is less studied than other types of pulmonary nodules (e.g., solid-type nodule). Difficulty in segmentation of GGO nodules is one of technical bottleneck in the development of CAD of GGO nodules. In this study, we propose an automated volumetric segmentation method for PNGGO using a modeling of ROI histogram with a Gaussian mixture. Our proposed method segments lungs and applies noise-filtering in the pre-processing step. And then, histogram of selected ROI is modeled as a mixture of two Gaussians representing lung parenchyma and GGO tissues. The GGO nodule is then segmented by region-growing technique that employs the histogram model as a probability density function of each pixel belonging to GGO nodule, followed by the elimination of vessel-like structure around the nodules using morphological image operations. Our results using a database of 26 cases indicate that the automated segmentation method have a promising potential.
\end{abstract}

Keywords: pure nodular ground-glass opacity, nodule segmentation, computer-aided diagnosis

\section{INTRODUCTION}

Recently, pure nodular ground-glass opacity (PNGGO) has been reported to increase among all types of CT-detected pulmonary nodules. A PNGGO, also referred to as a ground-glass opacity nodule is defined as hazy increased attenuation within the lung that does not obscure the bronchial and vascular margins on CT [1]. In the screening situations, PNGGOs have been reported to consist of 5.5-12\% of all CT-detected pulmonary nodules [2]. Some studies of pathologically proven cases have shown PNGGOs malignancy rates of $18 \%$ to $48 \%$ [3-5]; it is shown that, PNGGOs has considerable proportion of benign diseases. Therefore accurate estimation between malignant and benign lesions is important in order to reduce the number of unnecessary surgeries [2].

According to the early reports[4, 6], computerized quantification method was useful for differentiation of PNGGO. Reliable segmentation is one of the primary steps in computer aided diagnosis systems. However, segmentation techniques for PNGGO have rarely been reported compared to solid-type nodules [7-10]. The shape, pattern and boundary of PNGGO are very different from solid nodules. Furthermore, PNGGOs typically have ill-defined border and their feeding vascular structures are much more conspicuous than in case of solid nodules (see Figure 1 for an example PNGGO). Thus, segmentation methods commonly used for solid nodules do not fit in the segmentation task for PNGGOs.

In this research, we propose an automated volumetric segmentation method specially designed for PNGGO. Our proposed method segments lungs and applies noise-filtering in the pre-processing step. And then, histogram of selected ROI is modeled as a mixture of two Gaussians representing lung parenchyma and GGO tissues. The GGO nodule is then segmented by region-growing technique that employs the histogram model as a probability density function of each pixel belonging to GGO nodule, followed by the elimination of vessel-like structure around the nodules using morphological image operations. Our results using a database of 26 cases indicate that the automated segmentation method have a promising potential.

*Jong Hyo Kim: E-mail: kimihyo@,snu.ac.kr, Telephone: +82-2-2072-1660

Medical Imaging 2010: Computer-Aided Diagnosis, edited by Nico Karssemeijer, Ronald M. Summers, Proc. of SPIE Vol. 7624, 76241P · C 2010 SPIE · CCC code: 1605-7422/10/\$18 · doi: 10.1117/12.844108 


\section{MATERIALS}

\subsection{Data Acquisition and CT Protocols}

From April 2003 to March 2008, one chest radiologist (C.M.P) with 8 years of chest CT experience reviewed the records of patients who has PNGGO and underwent thin-section chest CT scans for lung cancer screening. Of these selected cases, we included cases that met the following criteria: (1) PNGGOs smaller than $3 \mathrm{~cm}$ in size, (2) PNGGOs determined to be persistent on follow-up CT performed at least one month after the initial CT. Based on these criteria, 26 PNGGOs in 25 individuals were included in this study.

CT imaging was performed using one of four CT systems (LightSpeed Ultra, GE Medical Systems, Milwaukee, Wis.; Sensation 16, Siemens Medical Systems, Erlangen, Germany; Brilliance 64 and MX8000, Philips Medical Systems, Best, The Netherlands). CT parameters for thin-section helical CT were as follows: detector collimation, 1.0- $2.5 \mathrm{~mm}$; beam pitch, 0.75-1.75; reconstruction thickness, $1.0-3.0 \mathrm{~mm}$; reconstruction interval, $1.0-2.5 \mathrm{~mm}$; rotation time, $0.5-1.0$ $\mathrm{s}$; tube voltage, $120 \mathrm{kVp}$; and tube current, $150-200 \mathrm{~mA}$, a reconstruction kernel and a high frequency algorithm were used.



Figure. 1. An example of pure nodular ground-glass opacity in high-resolution CT.

\section{METHODS}

Our proposed method consists of four steps, as shown in Figure 2, including pre-processing, histogram modeling, nodule segmentation and vessel-like structure removal.

\subsection{Pre-Processing}

The objective of the pre-processing step is to remove quantum noises within the ROI and to remove soft tissues outside the lung which is necessary for the ROIs drawn near the lung boundary (for juxtapleural nodule segmentation). A median filter was used for noise filtering, and simple thresholding (above -204 HU) was used for removal of soft tissues.

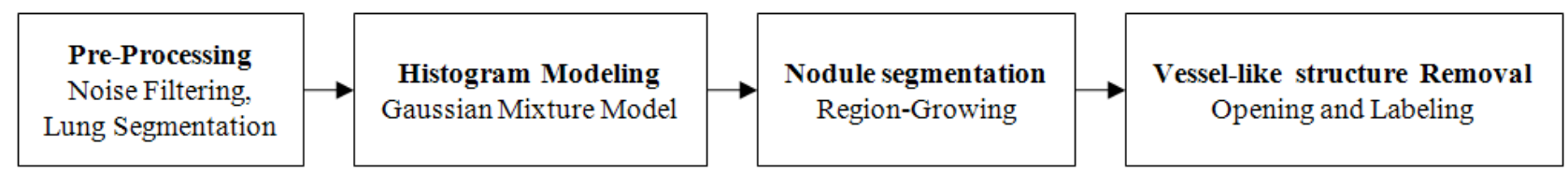

Figure 2. Flowchart of proposed ground-glass opacity segmentation method. 


\subsection{Histogram modeling}

Because of hazy attenuation pattern in ground-glass opacity and subtle differences of CT number between normal tissues, determining an appropriate threshold value for segmentation of GGO nodules is particularly challenging. We propose a histogram modeling approach for automated selection of the threshold value between ground-glass opacity and surrounding normal lung tissue using a Gaussian mixture model. A clustering algorithm estimates the probability density functions of the ground-glass opacity and normal tissue iteratively. The optimal threshold CT number was then automatically determined by a maximum likelihood estimation.

$$
\begin{aligned}
& p\left(x_{n} \mid \omega_{j}, \theta\right)=p\left(x_{n} \mid \mu_{j}, \sigma_{j}^{2}\right)=\frac{1}{\sqrt{2 \pi \sigma_{j}}} \exp \left(-\frac{\left(x_{n}-\mu_{j}\right)^{2}}{2 \sigma_{j}^{2}}\right) \\
& p\left(x_{n} \mid \theta\right)=\sum_{j=1}^{M} p\left(x_{n} \mid \mu_{j}, \sigma_{j}^{2}\right) \alpha_{j} \\
& \frac{\partial}{\partial \mu_{j}}[\cdot]=0, \quad \mu_{j}=\frac{\sum_{n=1}^{N} P\left(\omega_{j} \mid x_{n}, \theta\right) x_{n}}{\sum_{n=1}^{N} P\left(\omega_{j} \mid x_{n}, \theta\right)} \\
& \frac{\partial}{\partial \sigma_{j}}[\cdot]=0, \quad \hat{\sigma}_{j}^{2}=\frac{1}{d} \frac{\sum_{n=1}^{N} P\left(\omega_{j} \mid x_{n}\right)\left\|x_{n}-\hat{\mu}_{j}\right\|^{2}}{\sum_{n=1}^{N} P\left(\omega_{j} \mid x_{n}\right)} \\
& \frac{\partial}{\partial a_{j}}[\cdot]=0, \quad \hat{a}_{j}=\hat{P}\left(\omega_{j}\right)=\frac{1}{N} \sum_{n=1}^{N} P\left(\omega_{j} \mid x_{n}\right)
\end{aligned}
$$

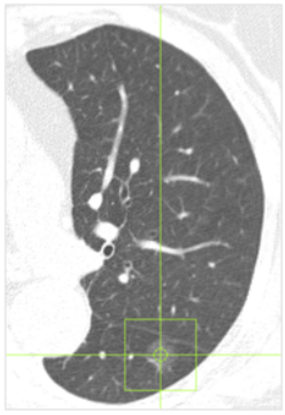

a)

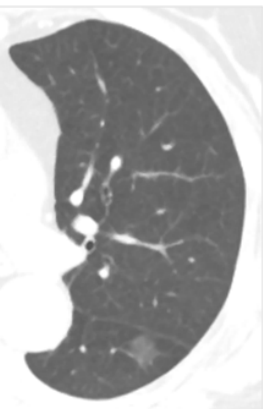

b)

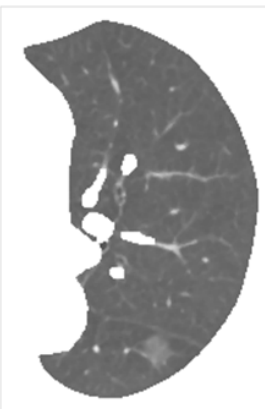

c)

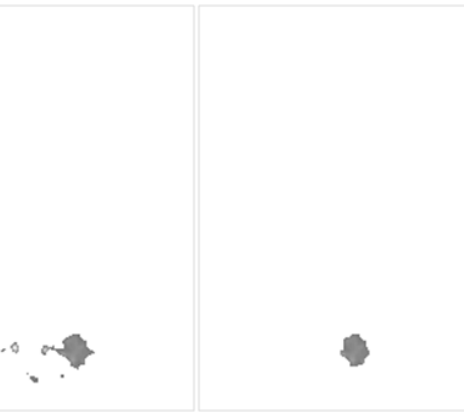

e)

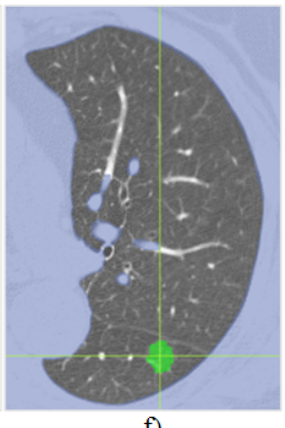

f)

Figure 3. An example segmentation procedure: (a) the original image, (b) after median filtering, (c) after lung tissue segmentation, (d) segmented lesion by 3D region-growing and histogram modeling (e) removal of vessel-like structure by $3 \mathrm{D}$ morphological opening operation, (f) the final segmentation image. 




Figure 4. a) histogram of an ROI including PNGGO, b) estimated probability density functions of lung parenchyma and PNGGO

\subsection{Region-growing segmentation}

After histogram modeling, a region-growing technique is applied within the user specified ROI. The center of ROI was used as a seed point, and the threshold value was used to limit growing of the regions. Thus, the connected voxels having intensity values higher than the threshold value were captured as a candidate GGO nodule in this stage.

\subsection{Vessel-like structure removal}

Finally, morphological opening and labeling operations were applied to remove the unnecessary linear structures attached to GGO nodules which often results from region-growing segmentation step. The opening operation removes the attaching linear structures, and the following labeling technique removes small islands. Figure 3 (e) illustrates this procedure.

\subsection{Evaluation of the proposed segmentation method}

For quantitative evaluation of proposed segmentation method, we compared the segmentation results of the automated method with the segmentation results manually edited by a radiologist. For all 26 PNGGOs, a representative slice was selected that showed largest section nodule, and the segmentation result was given as a semi-transparent color overlay on top of CT image. Then, one radiologist (C.M.P) manually edited the segmentation result on a pixel-by-pixel basis. The added and removed pixels were painted in different colors (red for added, blue for removed).

After manual editing, segmentation accuracy of the proposed method was evaluated using the edited segmentation as a gold standard. The Hausdorff distance, Tannimoto coefficient and Dice similarity coefficient were used for this evaluation $[11,12]$. The Hausdorff distance (HD) is a shape comparison metric based on binary image. The low Hausdorff Distance indicates high accuracy. The high Tannimoto coefficient and Dice similarity coefficient indicate high accuracy.

$$
\begin{aligned}
& \text { TannimotoCoefficient }=\frac{N(A \cap B)}{N(A \cup B)} \\
& \text { DiceSimilarityCoefficient }=\frac{2 N(A \cap B)}{N(A)+N(B)} \\
& \text { HausdorffDistance }=\max _{\sigma \in A}\left[\begin{array}{c}
\min _{\sigma \in B}[d(a, b)] \\
\sigma
\end{array}\right]
\end{aligned}
$$




\section{RESULTS}

The results of segmentation accuracy using the Hausdorff distance, Tannimoto coefficient and Dice similarity coefficient are shown in Table 1.

Table 1. Segmentation performance of the proposed method. The mean and standard deviation of the three indices between the automatic and manual segmentation were described.

\begin{tabular}{cc}
\hline Performance Index & Mean (Standard deviation) \\
\hline Hausdorff Distance & $0.000105(0.000150)$ \\
Tannimoto Coefficient & $0.914693(0.064465)$ \\
Dice Similarity Coefficient & $0.954269(0.036365)$ \\
\hline
\end{tabular}

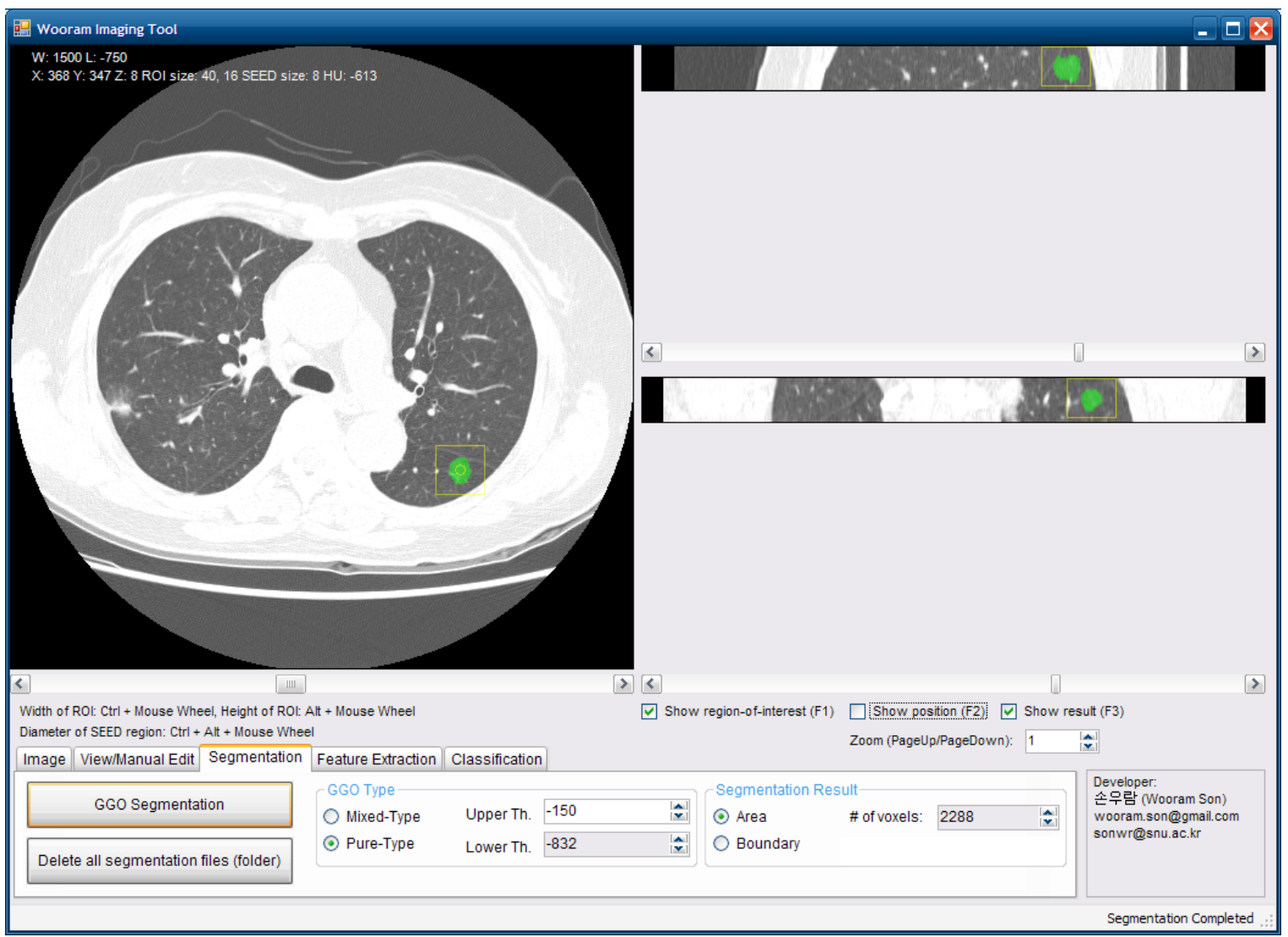

Figure 5. A screenshot of the developed PNGGO segmentation tool. 


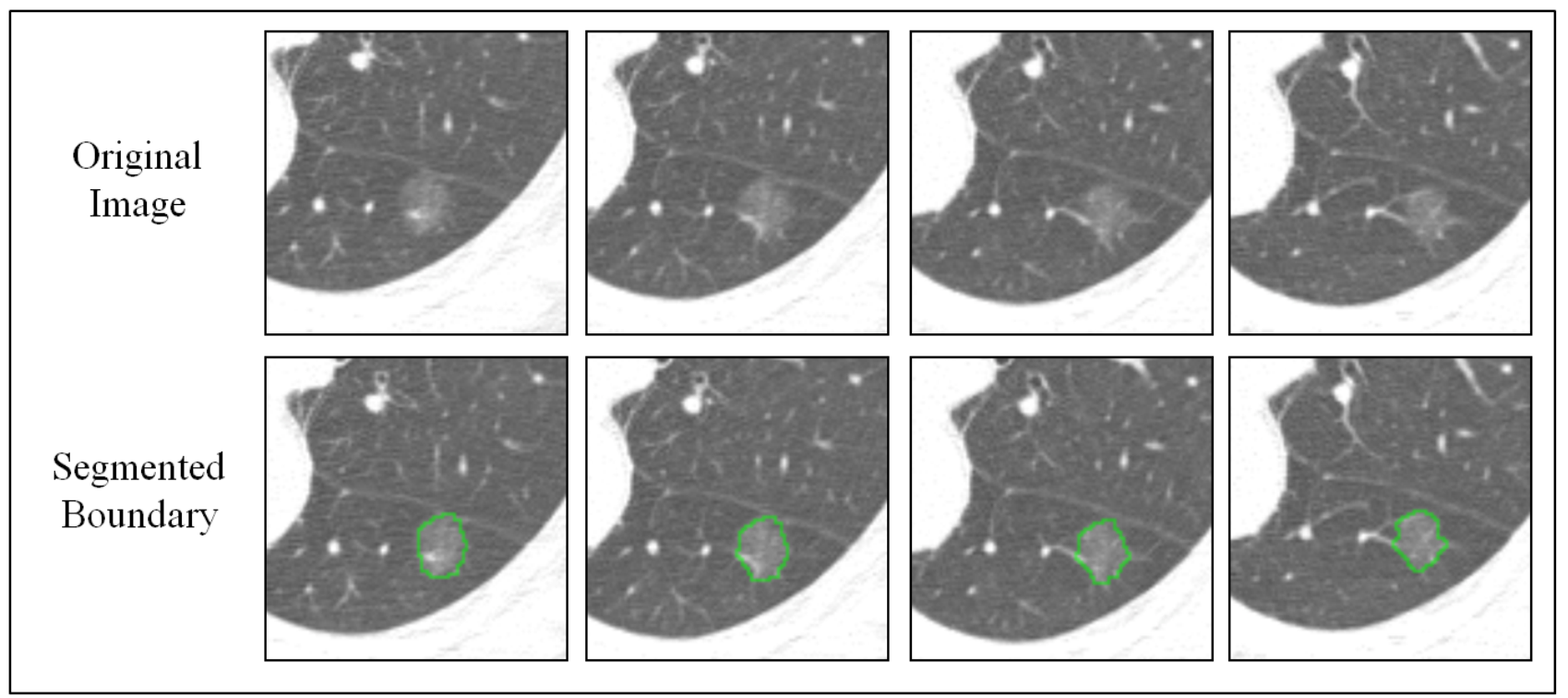

Figure 6. An example of PNGGO segmentation. The upper images are the largest slice CT image of the PNGGO. The segmentation results are in the lower images.

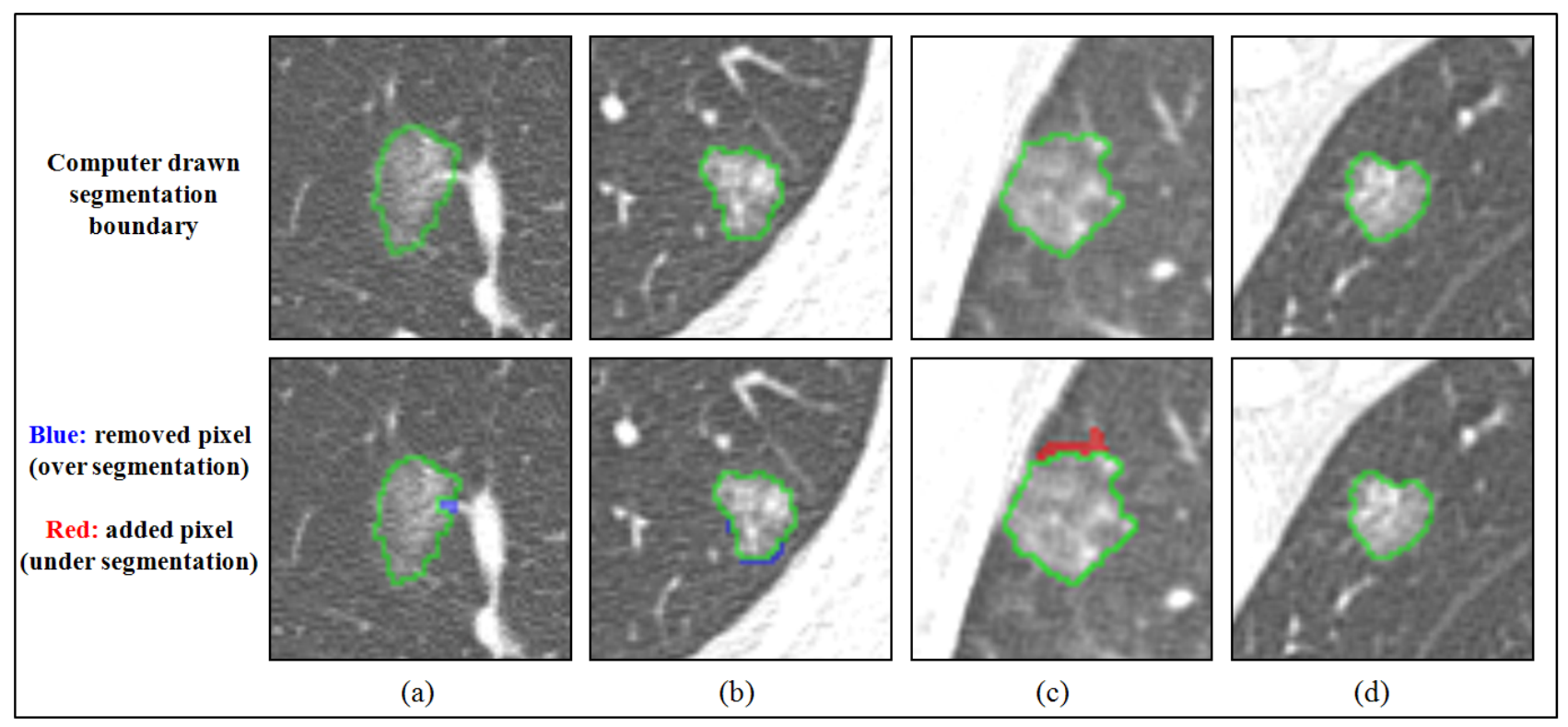

Figure 7. Automatic segmentation results are in the upper images. The lower images are comparing with manual segmentation performed by radiologist.

Table 2. The performance indices for the example 4 GGO nodules shown in figure 7.

\begin{tabular}{ccccc}
\hline Performance Index & Figure 7a & Figure 7b & Figure 7c & Figure 7d \\
\hline Hausdorff Distance & 0.000091 & 0.000070 & 0.000105 & 0.000 \\
Tannimoto Coefficient & 0.974874 & 0.942177 & 0.927959 & 1.000 \\
Dice Similarity Coefficient & 0.987277 & 0.970228 & 0.962633 & 1.000 \\
\hline
\end{tabular}




\section{CONCLUSIONS AND FUTURE WORK}

We have introduced a novel segmentation scheme specially tuned for automatic segmentation of PNGGOs based on histogram modeling and region-growing. Our segmentation scheme was applied to $25 \mathrm{CT}$ volume images including 26 GGO nodules. The results of segmentation of PNGGOs have been assessed quantitatively in comparison with manual segmentation performed by a radiologist. The mean Hausdorff distance was as low as 0.000105 , and Tannimoto and Dice similarity coefficients were 0.91 and 0.95 , respectively. These results show that our proposed method has promising potential for reliable segmentation of PNGGOs. We believe this work laid a ground for the development of CAD system both for detection and classification of PNGGOs. Future work will focus on further evaluation with larger database and multiple readers, and also extended application to mixed-type ground-glass nodules.

\section{REFERENCES}

1. J. Austin, N. Muller, P. Friedman et al., "Glossary of terms for CT of the lungs: recommendations of the Nomenclature Committee of the Fleischner Society," Radiology, 200(2), 327 (1996).

2. H. Lee, J. Goo, C. Lee et al., "Predictive CT findings of malignancy in ground-glass nodules on thin-section chest CT: the effects on radiologist performance," Eur Radiol, 19(3), 552-60 (2008).

3. R. Nakajima, T. Yokose, R. Kakinuma et al., "Localized Pure Ground-Glass Opacity on High-Resolution CT: Histologic Characteristics," J Comput Assist Tomogr, 26(3), 323-9 (2002).

4. K. Ikeda, K. Awai, T. Mori et al., "Differential Diagnosis of Ground-Glass Opacity Nodules: CT Number Analysis by Three-Dimensional Computerized Quantification," Chest, 132(3), 984-90 (2007).

5. H. Nomori, T. Ohtsuka, T. Naruke et al., "Differentiating between atypical adenomatous hyperplasia and bronchioloalveolar carcinoma using the computed tomography number histogram," Ann Thorac Surg, 76(3), 867871 (2003).

6. W. Son, S. Park, C. Park et al., "Classification of Benign/Malignant PNGGOs using K-means algorithm in MDCT Images: A Preliminary Study," IEICE Tech. Rep., 108(385), 257-260 (2009).

7. S. Sun, H. Li, X. Hou et al., "Automatic Segmentation of Pulmonary Nodules in CT Images." ICBBE, 790-793 (2007)

8. N. Xu, N. Ahuja, and R. Bansal, "Automated lung nodule segmentation using dynamic programming and EM based classification," Proc. SPIE, 4684, 666-676 (2002)

9. D. Yankelevitz, A. Reeves, W. Kostis et al., "Small pulmonary nodules: volumetrically determined growth rates based on CT evaluation," Radiology, 217(1), 251-6 (2000).

10. W. Browder, A. Reeves, T. Apananosovich et al., "Automated volumetric segmentation method for growth consistency of nonsolid pulmonary nodules in high-resolution CT," Proc. SPIE, 6514, 65140Y1-10 (2007)

11. S. Theodoridis, and K. Koutroumbas, [Pattern Recognition. 1999] Academic Press (1999).

12. D. Huttenlocher, G. Klanderman, and W. Rucklidge, "Comparing images using the Hausdorff distance," IEEE Trans. Pattern Anal. Machine Intell., 15(9), 850-863 (1993). 